\title{
Longstreth et al.'s reaction time model: Some comments
}

\author{
TARALD O. KVÅLSETH \\ University of Minnesota, Minneapolis, Minnesota
}

\begin{abstract}
Longstreth and his colleagues (Longstreth, 1988; Longstreth \& Alcorn, 1987; Longstreth, ElZahhar, \& Alcorn, 1985) have recently introduced a choice reaction time model based on a simple and novel behavioral explanation. However, as pointed out in this paper, there are several issues and problems associated with their model. In addition, it is shown that the reaction-time equation by Longstreth and his colleagues represents a special case of the general power law proposed earlier by this author. The type of behavioral explanation used by Longstreth and his colleagues is extended to the case of unequal stimulus probabilities. An alternative model is also developed using the repetition effect as a starting point.
\end{abstract}

For choice-reaction tasks with one-to-one stimulusresponse mapping, Longstreth and his colleagues (Longstreth, 1988; Longstreth \& Alcorn, 1987; Longstreth, ElZahhar, \& Alcorn, 1985) have formulated and discussed the model

$$
\mathrm{RT}=a+b\left(1-n^{-1}\right)
$$

as a replacement for the classical Hick-Hyman law (Hick, 1952; Hyman, 1953). In Equation 1, RT denotes the (mean) reaction time, $n$ is the number of stimulus-response (S-R) pairs, and $a$ and $b$ are constant parameters, both unrelated to $n$. Longstreth and his colleagues derived this RT equation from an attractively simple behavioral model.

However, a closer examination of the formulation and underlying explanations of the model reveals a number of questions and concerns with potential implications for the model's validity. Welford (1987) has raised some important issues. It is the purpose of this paper to elaborate briefly on a couple of Welford's points and to raise some additional ones. Longstreth and his colleagues' model considerations will be extended to the case of unequal stimulus probabilities, and some alternative model formulations and behavioral explanations will be offered. It will be assumed throughout this paper that other variables, such as error rates, are either negligible or approximately fixed by means of appropriate controls.

\section{COMMENTS}

\section{Is Hick-Hyman's Law False?}

Longstreth and his colleagues have argued that the classical Hick-Hyman law is false and should be replaced by their law (Equation 1). Their argument is based on the empirical observation that, for some experimental data sets, the RT versus $\log _{2} n$ or $\log _{2}(n+1)$ relationship tends

Address correspondence to Tarald $O$. Kvålseth, Department of Mechanical Engineering, University of Minnesota, Minneapolis, MN 55455 . to be curvilinear and negatively accelerated (convex upward), rather than linear as predicted by Hick-Hyman's law. Longstreth and his colleagues claim that, in a number of cases, Equation 1 provides more accurate fits to the experimental data than does Hick-Hyman's law. However, their proposition that Hick-Hyman's law should be rejected on the basis of such empirical evidence is too categorical. Welford (1987) has expressed the same opinion in defense of Hick-Hyman's law. In fact, Welford presented reanalyses of 18 previously published data sets for which Hick-Hyman's law provided superior fits than did those of Equation 1. Although, on purely empirical grounds, Hick-Hyman's law may not be uniformly superior to other lawful relationships, it has been clearly established that it does provide a good summary description of a substantial amount of data.

\section{Explanatory Model}

Rather than being empirically based, Longstreth and his colleagues' model is unique in that it has a very simple behavioral explanation. According to their explanatory model, the additional RT required as $n$ increases beyond $n=1$ is due to the subject having to switch his or her focal attention from one S-R element to the S-R element that occurs during a given experimental trial. This additional RT equals a constant time $b$ per attention switch multiplied by the probability $\left(1-n^{-1}\right)$ of the subject having to make an attention switch for any given stimulus occurrence. Equation 1 is obtained if we add another constant time component $a$ to the variable component $b\left(1-n^{-1}\right)$.

This underlying model assumes that the subject's attention is focused on only one S-R element at any given time. It also assumes that, when a stimulus occurs, at most one attention switch is made before the subject executes the response. However, the validity of these assumptions may be questioned. In particular, the assumption that at most one attention switch is made for each S-R occurrence would seem to be too strong and does not allow 
for any search among the elements of the response set. By comparison, other RT models assume elaborate search processes, both hierarchical and serial (see, e.g., Welford, 1976; Wickens, 1984).

\section{Erroneous Model Derivation}

Although it does not affect Equation 1 per se, it should be pointed out that Longstreth et al. (1985) made a rather obvious error when deriving Equation 1 . They started off with $b[E(E-1) / E]$ as denoting the average time required to shift attention to the target-code element, with $b$ being the time constant per switching operation and $E$ being the number of S-R elements in the code for a given set size (using the authors' terminology). Then, for $E=n$, they gave $b[n(n-1) / n]$ and stated that this expression simplifies to $b\left(1-n^{-1}\right)$. Of course, these terms become $b(E-1)$ and $b(n-1)$, and not $b\left(1-n^{-1}\right)$.

\section{Intercept $a$}

Welford (1987) pointed out that, for some data sets, the parameter $a$ in Equation 1 may be negative. Longstreth and Alcorn (1987) responded by saying that "for theoretical reasons, simple reaction time is not germane to the issue" (p. 315). However, Welford did not say that Equation 1 may predict negative simple reaction time; he said that the intercept parameter $a$ may be negative, and identified two particular data sets for which the estimate of $a$ turns out to be negative. Moreover, Longstreth and his colleagues treat the troublesome issue of a possible negative $a$ too lightly. They define $a$ as representing all RT components other than the component associated with $n$. Irrespective of whether simple RT is considered (1) equal to $a$, (2) as a component of $a$, or (3) irrelevant to the RT model altogether, the fact that $a$ may be negative is a problematical issue with relevance to the general validity of Equation 1 and its explanation.

\section{Power Law}

It may also be pointed out that Equation 1 is a special case of the power law

$$
\mathrm{RT}=a+b n^{\mathrm{c}}
$$

proposed by Kvålseth (1980). In this equation, $a, b$, and $c$ are the parameters, with $b>0$ for $c>0$, and $b<0$ for $c<0$. This fact is easily seen by introducing the parameters $\alpha$ and $\beta$ and expressing Equation 1 in the alternative form

$$
\mathrm{RT}=\alpha-\beta n^{-1} .
$$

For $b=-\beta$ and $c=-1$, Equation 2 reduces to Equation 1'. Of course, Longstreth and his colleagues' hyperbolic model in Equation 1', which has one less unknown parameter than Equation 2, cannot achieve the same goodness of fit to experimental data as the power model in Equation 2.

The power law in Equation 2 has been found to provide excellent fits to experimental data, even when the parameter $a$ is ignored (i.e., $a=0)$. For example, based on the data by Hilgendorf (1966), with $n=2,4,10,26$, 100 , and 1,000, Kvålseth (1980) found that RT = $.952 n^{.149}$ with a coefficient of determination $R^{2}=.993$, as compared to $R^{2}=.976$ and $R^{2}=.533$ as reported by Welford (1987) for Hick-Hyman's logarithmic model and for Longstreth and his colleagues' hyperbolic model, respectively. However, the power law expressed by Equation 2 is empirically based, and no behavioral explanation has so far been offered.

\section{Unequal Stimulus Probabilities}

The model by Longstreth and his colleagues was developed for the case in which the $n$ stimuli had the same probability of occurring in any trial. We shall now extend their model to the case with unequal stimulus probabilities $p_{1}, \ldots, p_{n}$ where $\sum_{i=1}^{n} p_{i}=1$. Three different attention strategies will be considered. First, consider that the probabilities with which the subject focuses attention on the individual S-R elements are the same as the probabilities of the elements occurring. That is, the probability is $p_{i}$ that the subject will focus on S-R element $i$, and $p_{i}$ is also the probability of element $i$ occurring, for $i=1, \ldots, n$. The joint probability that element $i$ will occur and that the subject will focus attention on element $i$ is $p_{i}^{2}$, since the event that element $i$ will occur and the event that the subject's focal attention will be on element $i$ are independent. The sum $\sum_{i=1}^{n} p_{i}^{2}$ becomes the probability that the subject will focus attention on the S-R element that occurs. Thus, the complement $1-\sum_{i=1}^{n} p_{i}^{2}$ is the probability that the subject has to switch attention from one S-R element to the element that does occur. If the time required for each attention-switching operation is $b$, and if another time constant $a$ is included as in Equation 1, we get the following RT model

$$
\mathrm{RT}=a+b\left(1-\sum_{i=1}^{n} p_{i}^{2}\right)
$$

For the case in which $p_{1}=\ldots=p_{n}=1 / n$, Equation 3 reduces to Equation 1.

Second, consider the strategy that the subject pays equal attention to the $n$ S-R elements. That is, the probability is $n^{-1}$ that the subject will focus attention on element $i$, whereas the actual probability of element $i$ occurring is $p_{i}$, for $i=1, \ldots, n$. By using the same probabilistic reasoning as in the preceding case, we see immediately that this equal-attention strategy leads to Equation 1.

As a third strategy, we may consider that the subject's focal attention is directed exclusively at the most likely S-R element, the probability of occurrence of which is $p_{\max }=\max \left\{p_{i}\right\}$. If two or more $p_{i} s$ are equal to $p_{\max }$, the subject is assumed to focus attention on the corresponding S-R elements with equal probability. Since the probability of the subject having to switch attention when an S-R element occurs is $1-p_{\max }$, and if $b$ is again the time taken per attention switch and $a$ is some overall time 
constant that is independent of the $p_{i} \mathrm{~s}$ and $n$, we get the model

$$
\mathrm{RT}=a+b\left(1-p_{\max }\right) .
$$

This model also leads to Equation 1 for the particular case when $p_{1}=\ldots=p_{n}=1 / n$.

We are not postulating that the models in Equations 3 and 4 are necessarily "good" choice RT models when the $p_{i} \mathrm{~s}$ are independent variables for a fixed $n$ or when the $p_{i} \mathrm{~s}$ and $n$ are simultaneously varied. However, these two mathematical models do result from the same type of underlying behavioral process as that considered by Longstreth and his colleagues when it is generalized to include the case with unequal stimulus probabilities.

\section{Repetition Effect}

A number of studies have demonstrated that the repetition of an S-R element produces a shorter reaction time to the second stimulus occurrence than does a nonrepetition, especially in a rapid series of stimulus presentations (see, e.g., Welford, 1976; Wickens, 1984). Whenever a nonrepetition occurs, time is required by the subject to identify the different stimulus (i.e., different from the immediately preceding one) and to select the appropriate response. Let this time be denoted by $b$, which may be considered to be constant for a fixed $n$. If stimulus $i$ occurs with the probability $p_{i}, i=1, \ldots, n$, then $1-\sum_{i=1}^{n} p_{i}^{2}$ is the probability of nonrepetition (i.e., the probability that the same stimulus will not occur twice in a row). The quantity $b\left(1-\sum_{i=1}^{n} p_{i}^{2}\right)$, then, is the choice RT component that accounts for stimulus identification and response selection for $n \geq 2$ and fixed. For each individual stimulus and response, and independent of the $p_{i} \mathrm{~s}$ and $n$, some constant time $a$ is required for such behavioral processes as stimulus detection and perception (encoding), neural signal transmission, brain recognition, signal decoding, and motorresponse execution, in addition to the possible time lags inherent in the experimental paradigm and apparatus. Thus, the total (mean) reaction time is given by

$$
\mathrm{RT}=a+b\left(1-\sum_{i=1}^{n} p_{i}^{2}\right)
$$

for a fixed $n$. For $n=1$, the term $b\left(1-\sum_{i=1}^{n} p_{i}^{2}\right)$ becomes zero and $\mathrm{RT}=a$.

We note that Equations 5 and 3 are identical, although their behavioral derivations are not identical. For Equation 3, and also for its special case of Equation 1, it has been assumed that the attention-switching time $b$ is unrelated to, or independent of, $n$. This assumption, which has not been made for the time parameter $b$ in Equation 5 , would seem to be of questionable validity. It would appear more reasonable to assume that attention-switching time would tend to increase as the number of S-R alternatives $n$ increases.

\section{REFERENCES}

HICK, W. E. (1952). On the rate of gain of information. Quarterly Journal of Experimental Psychology, 4, 11-26.

HILGENDORF, L. (1966). Information input and response time. Ergonomics, 9, 31-37.

HymaN, R. (1953). Stimulus information as a determinant of reaction time. Journal of Experimental Psychology, 45, 188-196.

KvÁLSETH, T. O. (1980). An alternative to the Hick-Hyman's and Sternberg's laws. Perceptual \& Motor Skills, 50, 1281-1282.

LONGSTRETH, L. E. (1988). Hick's law: Its limit is 3 bits. Bulletin of the Psychonomic Society, 26, 8-10.

Longstreth, L. E., \& AlCorn, M. B. (1987). Hick's law versus a power law: Reply to Welford. Journal of Experimental Psychology: General, 116, 315-316.

Longstreth, L. E., El-Zahhar, N., \& Alcorn, M. B. (1985). Exceptions to Hick's law: Explorations with a response duration measure. Journal of Experimental Psychology: General, 114, 417-434.

Welford, A. T. (1976). Skilled performance: Perceptual and motor skills. Glenview, IL: Scott Foresman.

Welford, A. T. (1987). Comment on "Exceptions to Hick's law: Explorations with a response duration measure." Journal of Experimental Psychology: General, 116, 312-314.

WICKENS, C. D. (1984). Engineering psychology and human performance. Columbus, $\mathrm{OH}$ : Merrill.

(Manuscript received December 14, 1988.) 